\title{
BMJ Open Congestive heart failure adherence redesign trial: a pilot study
}

\author{
Ashvarya Mangla, ${ }^{1,2}$ Rami Doukky, ${ }^{1,3,4}$ Lynda H Powell, ${ }^{1}$ Elizabeth Avery, ${ }^{1}$ \\ DeJuran Richardson, ${ }^{1,5}$ James E Calvin $\mathrm{Jr}^{6}$
}

To cite: Mangla A, Doukky R, Powell LH, et al. Congestive heart failure adherence redesign trial: a pilot study. BMJ Open 2014;4:e006542. doi:10.1136/bmjopen-2014006542

- Prepublication history for this paper is available online To view these files please visit the journal online (http://dx.doi.org/10.1136/ bmjopen-2014-006542).

Received 13 September 2014 Revised 24 October 2014 Accepted 30 October 2014

CrossMark

\footnotetext{
${ }^{1}$ Department of Preventive Medicine, Rush University Medical Center, Chicago, Illinois, USA

${ }^{2}$ Department of Internal Medicine, OSF St. Francis Medical Center, Peoria, Illinois, USA

${ }^{3}$ Division of Cardiology, John H. Stroger, Jr. Hospital of Cook County, Chicago, Illinois, USA

${ }^{4}$ Division of Cardiology, Rush University Medical Center, Chicago, Illinois, USA

${ }^{5}$ Department of Mathematics, Lake Forest College, Lake Forest, Illinois, USA

${ }^{6}$ Department of Medicine, University of Western Ontario, London, Ontario, Canada
}

Correspondence to Dr Rami Doukky; rami_doukky@rush.edu

\section{ABSTRACT}

Objective: Heart failure (HF) continues to be a leading cause of hospital admissions, particularly in underserved patients. We hypothesised that providing individualised self-management support to patients and feedback on use of evidence-based HF therapies (EBT) to physicians could lead to improvements in care and decrease hospitalisations. To assess the feasibility of conducting a larger trial testing the efficacy of this dual-level intervention, we conducted the Congestive Heart failure Adherence Redesign Trial Pilot (CHART-P), a proof-ofconcept, quasi-experimental, feasibility pilot study. Setting: A large tertiary care medical centre in Chicago. Participants: Low-income patients (<US\$30 000/year) hospitalised for exacerbation of systolic HF (ejection fraction $\leq 50 \%$ ) and their physicians. Twenty physicians and 33 patients were enrolled, of whom 23 patients completed the study.

Interventions: Physicians received HF guidelines and periodic individualised feedback on their adherence to EBT. Patients received HF education, support and selfmanagement training for diet and medication adherence by a trained nurse through 11 interactive sessions over a 4-month period. Evaluations were conducted preenrolment and 1 month postintervention completion.

Outcome measures: Feasibility was assessed by the ability to deliver intervention to patients and physicians. Exploratory outcomes included changes in medication and sodium intake for patients and adherence to EBT for physicians.

Results: Eighty-seven per cent and $82 \%$ of patients received $>80 \%$ of interventions at 1 month and by study completion, respectively. Median sodium intake declined ( 3.5 vs $2.0 \mathrm{~g} ; p<0.01$ ). There was no statistically significant change in medication adherence based on electronic pill cap monitoring or the Morisky Medication Adherence Scale (MMAS); however, there was a trend towards improved adherence based on MMAS. All physicians received timely intervention.

Conclusions: This pilot study demonstrated that the protocol was feasible. It provided important insights about the need for intervention and the difficulties in treating patients with a variety of psychosocial problems that undercut their effective care.

\section{INTRODUCTION}

Heart failure (HF) continues to increase in prevalence with an enormous impact on

\section{Strengths and limitations of this study}

- The intervention studied is multilevel, in that it targets physicians and patients simultaneously. Such strategies are potentially more potent than those with a single focus.

- The intervention studied provided individualised feedback to patients and physicians.

- The targeted population is low-income patients with heart failure who are disproportionally affected by the heart failure epidemic. Few studies have targeted this population, and our pilot study helped us in gaining important insights into this challenging group of patients before conducting a larger trial.

- This is a pilot feasibility study performed at a single medical centre

- The sample size is small and the duration of follow-up was short.

mortality (approximately $50 \%$ at 5 years postdiagnosis), hospitalisations and cost of care (US $\$ 30.7$ billion in 2012). ${ }^{12}$ The prevalence of HF among those 18 years and older in the USA is projected to increase by $46 \%$ in the next 15 years, resulting in more than 8 million people with $\mathrm{HF}$ by $2030 .^{2}$ This reality has created a significant and increasing financial burden on the healthcare system. Although HF therapies exist with demonstrated benefits on mortality, morbidity and quality of life, ${ }^{3}$ these therapies are being underutilised. ${ }^{4}$ Racial minorities and socioeconomically disadvantaged patients have a higher prevalence of HF and higher readmission rates, ${ }^{6} 7$ thus contributing disproportionately to the HF epidemic.

There is a particular need to develop effective interventions targeting economically disadvantaged patients with $\mathrm{HF}^{8}$ Results from our previously published Heart failure Adherence and Retention Trial (HART) suggested that patients with an annual income <US\$30 000 might benefit from counselling to improve self-management skills as a means to lower mortality and HF hospitalisation rates. ${ }^{9}$ Since physician adherence to evidence-based therapy has been shown to be suboptimal, 
providing education to physicians could offer additional value. We hypothesised that the use of a dual-level intervention strategy, intervening simultaneously on patients and their physicians, would translate into significantly improved quality of care among low-income patients with $\mathrm{HF}$ and improve their outcomes. To assess the feasibility of conducting a large trial to study the efficacy of this duallevel strategy, we conducted the Congestive Heart failure Adherence Redesign Trial (CHART) pilot study.

\section{METHODS}

The CHART pilot study was a proof-of-concept, pre-post treatment group only design. The key objective was to assess the feasibility and potential impact of our duallevel intervention for low-income patients with $\mathrm{HF}$ and their physicians. We would deem the intervention feasible if we were able to achieve four objectives: (1) assess patient adherence to prescribed therapies and sodium restriction, (2) deliver the intervention to patients, (3) assess physician adherence to evidence-based $\mathrm{HF}$ therapy and (4) provide timely feedback to physicians.

\section{Recruitment}

The study targeted patients with systolic HF with selfreported annual household income $<\mathrm{US} \$ 30000$, as these patients are at high risk for adverse outcomes. ${ }^{79}$ All participants were recruited while hospitalised at the Rush University Medical Center in Chicago, Illinois. Patients were identified via monitoring of hospital admission logs and the echocardiography laboratory database. New HF admissions with systolic dysfunction (ejection fraction $\leq 50 \%$ as measured by echocardiography, radionuclide ventriculography or radiographic contrast ventriculography) were included. Patients having HF with preserved ejection fraction were excluded as there are no set guidelines for managing these patients, deeming the proposed physicianlevel intervention non-feasible. Eligibility of the identified candidates was then determined based on self-reported income. Exclusion criteria included being a cardiac transplant candidate, having severe aortic stenosis, uncontrolled ventricular arrhythmias, B-type natriuretic peptide $<100 \mathrm{pg} / \mathrm{mL}$, severe asthma or chronic obstructive pulmonary disease, major psychiatric comorbidities, alcohol or drug addiction, haemodialysis treatment, debilitating neurological conditions, severe arthritis, peripheral arterial disease, or having an uncertain 12 month prognosis. Once an eligible patient was identified, it was checked whether their physician was on staff at the medical centre. If the physician was on staff, their consent for study participation was obtained. Subsequently, the patients were recruited and consented. As this was a proof-of-concept study primarily aimed at assessing feasibility, sample size calculations were not performed.

\section{Interventions}

The dual-level intervention targeted both the patients and physicians caring for their HF (a general practitioner or a cardiologist). Physician-level intervention provided them with evidence-based HF guidelines in the form of a brochure summarising the 2005 American College of Cardiology/American Heart Association (ACC/AHA) recommendations with updates from the 2009 guidelines, which were current at the time of our study. ${ }^{10}{ }^{11}$ Physicians also received summary reports of their patient's medication use at baseline, 1 and 4 months, as obtained from medical records and patient self-reports. These reports were accompanied by standardised personal notes from our study cardiologist (JEC) outlining patient-specific recommendations, such as possible medication changes, that could optimise adherence to evidence-based therapy as defined by class I ACC/AHA recommendations. This being a feasibility study with a short follow-up time, we addressed only evidence-based medication classes but not optimal medication dosing. No feedback to the personal notes was solicited or tracked.

Patient-level intervention provided to each enrolled patient a tailored HF self-management kit. ${ }^{12}$ The kit included brochures featuring patient-friendly HF education material and a variety of self-management tools (scale, measuring bottle, educational materials and pillboxes). Patients were coached on use of the kit during one-on-one contacts timed to occur 3 days post hospital discharge, then once weekly for 1 month and every 2 weeks thereafter for another 3 months, totalling 11 intervention contacts. The patient contacts were performed in person or via telephone at the patient's discretion. These contacts were conducted by a trained nurse who followed a problem-solving format in which patients were encouraged to articulate barriers to their adherence and to use a variety of self-management strategies to overcome them. ${ }^{13}$ Simple metaphors were used to educate patients about their HF. The heart was equated to a 'workhorse' carrying a 'load' (blood and water) throughout the body via the 'road' (arteries). 'Water pills' (diuretics) 'lighten the load' and ACE inhibitors (ACE-I) or angiotensin receptor blockers (ARBs) 'widen the road', making it easier for the workhorse to pump. The importance of adhering to prescribed medications, a low sodium diet, daily weighing, regular physical activity, smoking cessation, and regular follow-up with providers was emphasised. Stress reduction strategies were also discussed. Patient comprehension of these components was assessed at each contact and their knowledge reinforced whenever gaps were identified.

\section{Outcomes, measures, and data analyses}

The impact of the dual-level intervention on physician adherence to evidence-based therapy focused specifically on appropriate use of an ACE-I or ARB, $\beta$-blocker and aldosterone antagonist. Data on physician adherence were gathered by chart audit at baseline and at 5 months for each patient enrolled. Only patients who remained in the study for the 5 month evaluation were included in these analyses. Physician adherence with respect to a given medication was measured using the 
proportion of their patients who were prescribed the medication, based on those for whom it was appropriate according to the 2005 and 2009 ACC/AHA guidelines and as confirmed by the study cardiologist. ${ }^{10}{ }^{11}$ Pre-post change in percentage physician adherence and average physician adherence was assessed via Student $t$ tests or the Wilcoxon tests, as appropriate.

Patient adherence to prescribed therapies was determined at baseline and at 5 months, using both electronic pill cap monitoring (using the MEMS V Trackcap; AARDEX, Zug, Switzerland) and the Morisky Medication Adherence Scale (MMAS). ${ }^{14}$ MMAS provides a score of $0-4$, with 4 indicating the highest adherence. Each patient's pill cap use was monitored for an ACE-I, ARB, $\beta$-blocker or diuretic, in that order depending on which of these drugs was prescribed. Patients were instructed to place a month's supply of monitored medication into their pill cap container and use it over the ensuing month. Patient adherence was then measured based on the percentage of time a patient took a pill relative to the prescribed timing. Patients were designated 'adherent' if their observed adherence was $\geq 80 \% .^{913} 15$ The pre-post change in per cent adherence was analysed via paired Student $\mathrm{t}$ tests or the Wilcoxon tests, as appropriate. The pre-post change in the proportion of patients designated as 'adherent' (via a pill cap or MMAS) was analysed via McNemar's exact test. Sodium intake was determined by a Food Frequency Questionnaire specifically designed to assess sodium intake ${ }^{16}$ and the pre-post change was analysed via paired Student $t$ test or the Wilcoxon test, as appropriate.

Sensitivity analyses were conducted to account for missing data at the 5-month data collection. The analysis consisted of a comparison of results under three different data replacement approaches: (1) a 'Best Case' scenario in which missing values were replaced with values indicating 'adherence' (the maximum value, for the MMAS); (2) a 'Worst Case' scenario in which missing values were replaced with values indicating 'non-adherence' (minimum value for MMAS) and (3) a 'Middle Case' scenario in which missing values were replaced with the last observation carried forward.

During scheduled follow-up visits, patients were asked whether or not they had been recently hospitalised. Data on all reported hospitalisations were collected after obtaining proper consent. To provide a preliminary estimate of the intervention's impact on rehospitalisations, the 30-day readmission rate among the study cohort was compared with the year 2010's 30-day hospital readmission rate at the site from which they were recruited (Rush University Medical Center).

\section{RESULTS}

Between January and July 2010; 266 patients with systolic HF were screened (figure 1); 146 met the exclusion criteria; 29 were unreachable; 22 patients refused to enrol and the physicians for 36 patients refused to participate in the study. Data on the reasons for physicians' refusal were not gathered. Thirty-three patients and their 20 physicians (18 cardiologists and 2 general practitioners) were enrolled; 23 patients $(70 \%)$ fully completed the study follow-up; 1 patient died $(3 \%)$ and $5(15 \%)$ withdrew. Four patients $(12 \%)$ were lost to follow-up after they had received $>80 \%$ of their intervention visits. There were no clinically or statistically significant differences in the baseline characteristics of patients who completed the study versus those who did not.

Table 1 summarises the baseline characteristics of all the 33 patients who were enrolled. Table 2 summarises the outcome data for physicians and patients at study completion. Physician adherence with respect to any given drug class could only be evaluated in patients with an indication for that medication at baseline and at 5 months. As a result, of the 20 physicians who were enrolled, only 13 were evaluable for ACE-I/ARBs, 10 for $\beta$-blockers, and 11 for aldosterone antagonists. At baseline, $69 \%$ and $100 \%$ of physicians were appropriately prescribing ACE-I/ARB and $\beta$-blockers, respectively, over all of their patients enrolled in the study. At 5 months, this adherence rate increased to $77 \%$ of enrolled physicians for ACE-I/ARBs and decreased to $90 \%$ for $\beta$-blockers. For aldosterone antagonists, physician adherence was $17 \%$ at baseline and dropped to $0 \%$ at 5 months. These changes in physician adherence rates were not statistically significant.

For the 23 patients who completed the study, selfreported sodium intake declined by $1192 \mathrm{mg}(\mathrm{p}<0.01)$ from baseline. Their pill cap adherence decreased as only 4 of $23(17 \%)$ patients were adherent at study completion versus 10 of $23(43 \%)$ at baseline $(p=0.02)$. However, the proportion of patients scoring the maximum on MMAS improved from $9(39.1 \%)$ at baseline to $15(65.2 \%)$ at study completion $(\mathrm{p}=0.18)$.

The feasibility of the protocol for patients was assessed as adherence to patient interventions: all patients received at least 1 intervention visit, $87 \%(29 / 33)$ of patients completed at least $4(80 \%)$ of the protocolrequired 5 scheduled intervention visits within the first month and $82 \%(27 / 33)$ of participants completed at least $9(81.8 \%)$ of the protocol-required 11 intervention visits within the 4-month intervention period. Among all completed visits, $66 \%$ were performed in person and the remaining by phone. Given the small sample size, it is difficult to comment on the differences in outcomes based on whether a patient received the majority of interventions in person versus over the phone. All physicians received the study-supplied patient summary report within 2 weeks of the data being collected from the patient.

The 33 patients reported a total of 20 hospitalisations during the study follow-up, for which medical records were reviewed. The 30-day all-cause readmission rate was $15.2 \%(5 / 33)$, all of which were for HF exacerbation. The 30-day all-cause readmission rate for the hospital in 2010 was $28.3 \%(143 / 505)$. 
Figure 1 Flow sheet from screening to study completion.

Patients with systolic heart failure screened for study ( $\mathrm{N}=\mathbf{2 6 6})$

Excluded from study $(\mathrm{N}=\mathbf{2 3 3})$ :

- 146 met exclusion criteria.

- 29 patients were unreachable.

- 22 patients refused to participate.

- 36 patients' physicians refused to participate.

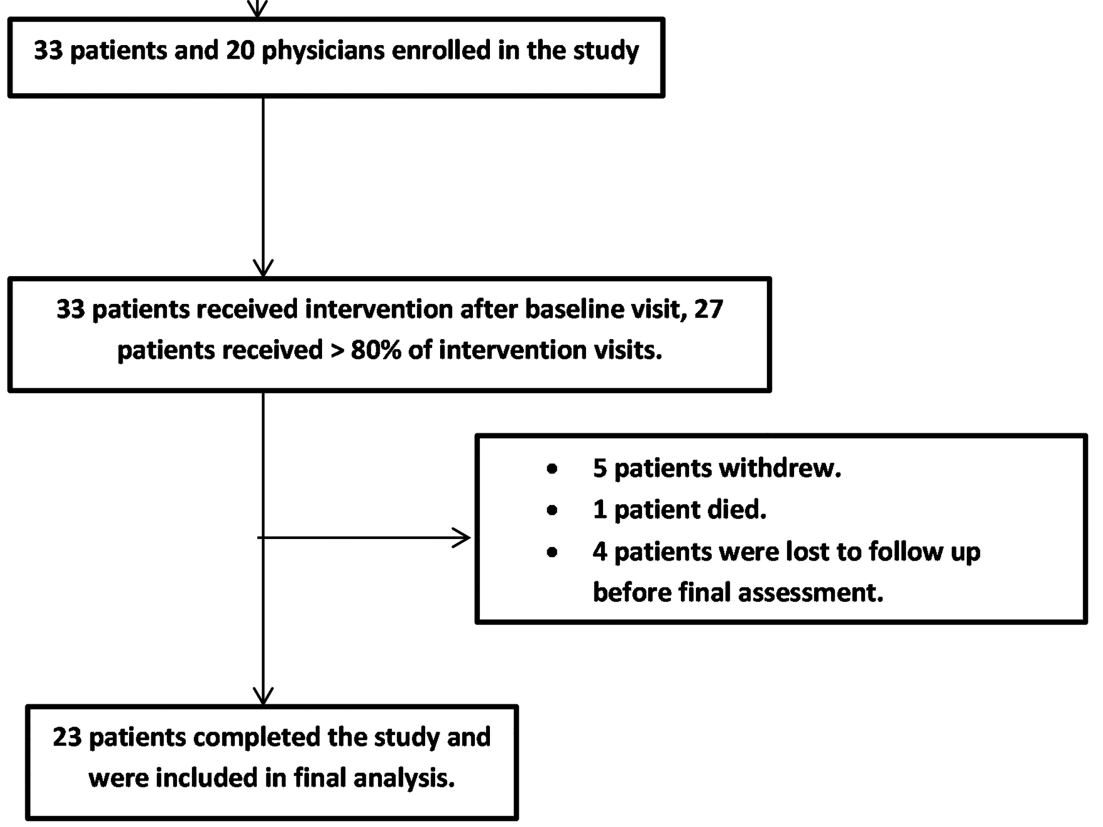

Sensitivity analyses with respect to missing data at the 5-month study visit revealed that the results presented in table 2 were fairly robust, given the size of the sample. Almost all changes from baseline in physician adherence continued to be non-statistically significant for all three medication classes under all three sensitivity analysis scenarios. The exception was observed under the 'Worst Case' scenario, in which an even deeper decrease in adherence with respect to $\beta$-blockers was observed that achieved statistical significance $(\mathrm{p}<0.01)$. Sensitivity analyses with respect to patient-drug adherence and sodium intake data produced results similar to those presented in table 2, with the exception of an even greater decrease (that was statistically significant, $\mathrm{p}<0.01)$ in patient adherence as measured by pill cap at 5 months under the 'Worst Case' scenario and a statistically significant $(p=0.03)$ increase in adherence as measured by the Morisky score under the 'Best Case' scenario.

\section{DISCUSSION}

The primary purpose of this pilot study was to assess the feasibility of a novel intervention and gain important insights into issues that may need to be addressed in a larger trial. ${ }^{17}$ From that perspective, our pilot study had encouraging results. We were able to deliver the intervention to both physicians and patients. All physicians received appropriate patient-specific feedback in a timely manner and more than $80 \%$ of patients completed at least $80 \%$ of their intervention visits. We were able to acquire data on hospitalisations and deaths. These findings suggest that the intervention is amenable to implementation in a larger trial. While a pilot study is not designed to provide definitive insights as to the impact of an intervention, we observed a significant decline in sodium intake and a trend towards a lower rate of $\mathrm{HF}$ hospitalisations in study patients compared with the general hospital census. One of the exploratory aims of our study was to assess changes in sodium intake, as sodium restriction in patients with $\mathrm{HF}$ was an area of potential intervention based on the prevailing literature at the time. ${ }^{18}{ }^{19}$ In fact, sodium restriction in patients with $\mathrm{HF}$ was a class I recommendation based on the 2005 and 2009 ACC/AHA guidelines. ${ }^{10} 11$ This has been challenged recently by studies showing adverse outcomes in patients with $\mathrm{HF}$ with low daily sodium intake. $^{20-22}$ Current ACC/AHA guidelines for HF still recommend sodium restriction, though as a Class IIa recommendation. ${ }^{3}$ 
Table 1 Baseline characteristics of enrolled patients

\begin{tabular}{|c|c|}
\hline Total enrolled & 33 \\
\hline \multicolumn{2}{|l|}{ Demographics } \\
\hline Age (years), mean $\pm S D$ & $58 \pm 13.0$ \\
\hline Women, $\mathrm{N}(\%)$ & $21(63.6)$ \\
\hline African-American, N (\%) & $29(87.9)$ \\
\hline Hispanic, N (\%) & $3(9.1)$ \\
\hline \multicolumn{2}{|c|}{ Age at end of education (years), $\mathrm{N}(\%)$} \\
\hline $8-11$ & $10(30.3)$ \\
\hline 12 & $13(39.4)$ \\
\hline $13-15$ & $9(27.3)$ \\
\hline $17+$ & $1(3.0)$ \\
\hline \multicolumn{2}{|l|}{ Income, N (\%) } \\
\hline US\$0-US \$4999 & $2(6.1)$ \\
\hline US $\$ 5000-$ US $\$ 9999$ & $9(27.3)$ \\
\hline US\$10 000-US\$14 999 & $6(18.2)$ \\
\hline US\$15 000-US\$19 999 & $4(12.1)$ \\
\hline US\$20 000-US\$24 999 & $6(18.2)$ \\
\hline US\$25 000-US\$29999 & $6(18.2)$ \\
\hline \multicolumn{2}{|l|}{ Medical history, N (\%) } \\
\hline Current smoker & $3(9.1)$ \\
\hline Past smoker & $6(18.2)$ \\
\hline Has a pacemaker & $20(60.6)$ \\
\hline Valvular heart disease & $6(18.2)$ \\
\hline Hypertension & $31(93.9)$ \\
\hline Coronary artery disease & $19(57.6)$ \\
\hline Myocardial infarction & $7(21.2)$ \\
\hline Stroke & $4(12.1)$ \\
\hline Diabetes mellitus & $18(54.5)$ \\
\hline COPD $^{*}$ & $2(6.3)$ \\
\hline Asthma & $8(24.2)$ \\
\hline $\begin{array}{l}\text { Morisky Medication Adherence Scale } \\
\text { score of } 4 \text {; median }(Q 1, Q 3) \dagger\end{array}$ & $3(3,4)$ \\
\hline Serum Creatinine $(\mathrm{mg} / \mathrm{dL})$, mean $\pm \mathrm{SD}$ & $1.5 \pm 0.8$ \\
\hline Systolic BP $(\mathrm{mm} \mathrm{Hg})$, mean $\pm \mathrm{SD}$ & $116.6 \pm 16.7$ \\
\hline Diastolic BP $(\mathrm{mm} \mathrm{Hg})$, mean $\pm \mathrm{SD}$ & $74.1 \pm 11.7$ \\
\hline $\mathrm{BP}>130 / 80, \mathrm{~N}(\%)$ & $5(15.2)$ \\
\hline $\mathrm{BMI}\left(\mathrm{kg} / \mathrm{m}^{2}\right)$, mean $\pm \mathrm{SD}^{*}$ & $33.4 \pm 7.7$ \\
\hline Dietary sodium $(\mathrm{g})$, mean $\pm \mathrm{SD}^{*}$ & $3.6 \pm 1.5$ \\
\hline \multicolumn{2}{|l|}{ Patient medication adherence } \\
\hline Pill caps returned, $\mathrm{N}(\%)$ & $30(90.9)$ \\
\hline \multicolumn{2}{|l|}{ Medications measured, N (\%) } \\
\hline - ACE inhibitor & $19(57.6)$ \\
\hline Angiotensin receptor blocker & 9 (27.3) \\
\hline - $\beta$-blocker & $4(12.1)$ \\
\hline Diuretic & $1(3.0)$ \\
\hline $\begin{array}{l}\text { Percentage of prescribed drugs } \\
\text { taken, } \\
\text { median (Q1, Q3) }\end{array}$ & $77.3(55.4,88.9)$ \\
\hline $\begin{array}{l}\text { Participants } \geq 80 \% \text { adherent } \$ \text {, } \\
\mathrm{N}(\%)\end{array}$ & $13(43.3)$ \\
\hline \multicolumn{2}{|c|}{$\begin{array}{l}{ }^{*} \mathrm{~N}=32 . \\
+S c o r e \text { of } 4 \text { on Morisky Medication Adherence Scale indicates full } \\
\text { medication adherence. } \\
\mathrm{fN}=30 \text {. } \\
\mathrm{BMI} \text {, body mass index; BP, blood pressure; COPD, chronic } \\
\text { obstructive pulmonary disease. }\end{array}$} \\
\hline
\end{tabular}

Our pill cap data indicated a trend towards decreased patient adherence postintervention while data from MMAS indicated otherwise. Although pill cap monitoring is an objective adherence measure, it relies on appropriate use by the patient. MMAS, on the other hand, is subjective but has been shown to correlate with cardiovascular outcomes. ${ }^{23}$ This discordance between pill cap and MMAS data may have been due to poor acceptability of pill caps among study participants or due to biased reporting with MMAS, as patients may have learnt to provide favourable answers. Furthermore, $7(30 \%)$ participants who completed the study follow-up did not return their electronic pill caps at the study intervention, handicapping our ability to draw inferences concerning patient medication adherence and representing a large drawback to this methodology. Use of pill caps in low-income populations poses challenges. Future studies should initiate measures to ensure adequate patient education on their use and return.

Physician adherence to ACE-I/ARB and $\beta$-blockers was high at baseline with not much room for improvement. For aldosterone antagonists, the prescription rate was low at baseline. Aldosterone antagonists require careful and regular monitoring of renal function and serum potassium levels. Such rigorous monitoring may be difficult in the challenging patient population that we studied, and could account for the low adherence to a certain extent. In a larger trial, if we can ensure timely physician follow-up, the prescription of this class of medications to appropriate patients may see a better trend. Recruiting physicians was met with resistance, as some physicians were unwilling to participate. Our dual intervention strategy is relatively novel, and with this being a pilot study, resistance from physicians is not surprising. It is plausible that the physicians who refused to participate may represent a subset of providers who are not receptive to feedback. The impact of physicians' unwillingness to participate on patients' outcome remains unknown. Providing feedback to physicians regarding their adherence to evidence-based therapy is likely to be part of healthcare delivery going forward. These have been implemented in a variety of ways such as providing reimbursement incentives, penalties and electronic medical record alerts. In this trial, we chose to provide more personalised and patient-centred feedback. The ideal mechanism and format of most effective feedback needs to be investigated.

Approximately $27 \%$ of the enrolled patients withdrew or were lost to follow-up. Of these, two patients completed their interventions but did not return for the postintervention visit; four patients refused additional follow-up after the baseline visit. Our interactions with these participants revealed that they were experiencing a variety of social, psychological and emotional challenges that limited their ability to effectively manage their HF. Expanding the scope of the patient-level intervention to provide stronger and more personal support may enhance their ability to self-manage their condition. This could be achieved by utilising community health workers who are part of a patient's local community and can go directly to their homes, providing support and 
Table 2 Physician and patient adherence at baseline and 5 months

\begin{tabular}{|c|c|c|}
\hline & Baseline & 5 months \\
\hline Physician full adherence* & $\mathrm{N}=20$ & $\mathrm{~N}=20$ \\
\hline ACE-I/ARB, N/total (\%) & 9/13 (69.2) & 10/13 (76.9) \\
\hline$\beta$-blockers, N/total (\%) & $10 / 10(100)$ & $9 / 10(90)$ \\
\hline Aldosterone antagonists, N/total (\%) & $2 / 12(16.7)$ & $0 / 12(0.0)$ \\
\hline Patient adherence & $\mathrm{N}=33$ & $\mathrm{~N}=23$ \\
\hline Percentage of prescribed drugs takent, median (Q1, Q3) & $82.6(76.0,94.0)$ & $71.8(38.3,79.8)$ \\
\hline Participants $\geq 80 \%$ adherent $\dagger, \mathrm{N}(\%)$ & $10(43.5)$ & $4(17.4)$ \\
\hline Full adherence by MMASł, $\mathrm{N}(\%)$ & $9(39.1)$ & $15(65.2)$ \\
\hline Sodium intake $(\mathrm{mg})$, median $(\mathrm{Q} 1, \mathrm{Q} 3) \S$ & $3464(2400,4125)$ & $2036.5(1800,2384)$ \\
\hline
\end{tabular}

counselling in important psychosocial areas which undercut delivery of quality medical care. This can potentially lead to better outcomes in this challenging patient population.

\section{Lessons learnt}

Given our experiences in the CHART pilot trial, we implemented the following adjustments in the large CHART (NCT01698242): (1) we decided to approach the physicians before the patients, which streamlined the recruitment process and guided appropriate resource utilisation; (2) patients were screened more rigorously to avoid early dropouts; (3) we decided to provide the patient-level intervention at the patient's home, rather than at a clinic, utilising community health workers who are members of the patient's own community and can cross cultural and logistical barriers encountered in delivering care for low-income patients; (4) we implemented reminders and incentives to enhance the return of the pill cap bottles in order to ensure completeness of medication adherence data and (5) we enhanced the physician-level intervention by providing access to online educational modules and simplifying physician feedback to include graphically presented adherence data.

\section{CONCLUSION}

Dual-level interventions appear to provide a promising strategy for improving outcomes among low-income patients with HF. Our findings indicate not only potential benefits but also unique challenges in treating patients from disadvantaged backgrounds. Attention to psychosocial and logistical issues that undercut effective medical care may be needed.

Acknowledgements The authors would like to acknowledge the efforts of Claudia Eaton for helping with data collection, patient recruitment and patient intervention, and John Kane for helping with data analysis.

Contributors $\mathrm{AM}$ and RD contributed with literature review and manuscript preparation. AM assisted with data collection. EA and DR were responsible for data analysis and assisted with manuscript preparation. LHP and JEC were responsible for study design and conduct, and supervised manuscript preparation. JEC was responsible for physician intervention and assessing physician adherence to evidence-based therapy.

Funding Funding provided by Novartis (Basel, Switzerland).

Competing interests RD serves on the advisory board of Astellas Pharma US (Northbrook, Illinois, USA) and received research funding grants from Astellas Pharma, US.

Ethics approval Institutional Review Board at Rush University Medical Center Provenance and peer review Not commissioned; externally peer reviewed. Data sharing statement No additional data are available.

Open Access This is an Open Access article distributed in accordance with the Creative Commons Attribution Non Commercial (CC BY-NC 4.0) license, which permits others to distribute, remix, adapt, build upon this work noncommercially, and license their derivative works on different terms, provided the original work is properly cited and the use is non-commercial. See: http:// creativecommons.org/licenses/by-nc/4.0/

\section{REFERENCES}

1. Go AS, Mozaffarian D, Roger VL, et al. Heart disease and stroke statistics-2014 update: a report from the American Heart Association. Circulation 2014;129:e28-292.

2. Heidenreich PA, Albert NM, Allen LA, et al. Forecasting the impact of heart failure in the United States: a policy statement from the American Heart Association. Circ Heart Fail 2013;6:606-19.

3. Yancy CW, Jessup M, Bozkurt B, et al. 2013 ACCF/AHA guideline for the management of heart failure: a report of the American College of Cardiology Foundation/American Heart Association Task Force on Practice Guidelines. J Am Coll Cardiol 2013;62:e147-239.

4. Fonarow GC, Yancy CW, Hernandez AF, et al. Potential impact of optimal implementation of evidence-based heart failure therapies on mortality. Am Heart J 2011;161:1024-30.e3.

5. Calvin J, Shanbhag S, Avery E, et al. Adherence to evidence-based guidelines for heart failure in physicians and their patients: lessons from the Heart Failure Adherence Retention Trial (HART). Congest Heart Fail 2012;18:73-8.

6. Blair J, Huffman M, Shah S. Heart failure in North America. Curr Cardiol Rev 2013;9:128-46.

7. Hawkins NM, Jhund PS, McMurray JJ, et al. Heart failure and socioeconomic status: accumulating evidence of inequality. Eur $J$ Heart Fail 2012;14:138-46.

8. Macabasco-O'Connell A, Crawford MH, Stotts N, et al. Gender and racial differences in psychosocial factors of low-income patients with heart failure. Heart Lung 2010;39:2-11.

9. Powell LH, Calvin JE Jr, Richardson D, et al. Self-management counseling in patients with heart failure: the heart failure adherence and retention randomized behavioral trial. JAMA 2010;304:1331-8.

10. Hunt SA, Abraham WT, Chin MH, et al. ACC/AHA 2005 Guideline Update for the Diagnosis and Management of Chronic Heart Failure in the Adult: a report of the American College of Cardiology/ American Heart Association Task Force on Practice Guidelines 
(Writing Committee to Update the 2001 Guidelines for the Evaluation and Management of Heart Failure): developed in collaboration with the American College of Chest Physicians and the International Society for Heart and Lung Transplantation: endorsed by the Heart Rhythm Society. Circulation 2005;112:e154-235.

11. Hunt SA, Abraham WT, Chin MH, et al. 2009 focused update incorporated into the ACC/AHA 2005 Guidelines for the Diagnosis and Management of Heart Failure in Adults: a report of the American College of Cardiology Foundation/American Heart Association Task Force on Practice Guidelines: developed in collaboration with the International Society for Heart and Lung Transplantation. Circulation 2009;119:e391-479.

12. http://www.carekit.com/heartfailurekit.html. Buffalo Grove, IL, USA: Communication Science, Inc., 2009.

13. Powell LH, Calvin JE Jr, Mendes de Leon CF, et al. The Heart Failure Adherence and Retention Trial (HART): design and rationale. Am Heart J 2008;156:452-60.

14. Morisky DE, Green LW, Levine DM. Concurrent and predictive validity of a self-reported measure of medication adherence. Med Care 1986;24:67-74

15. Ho PM, Bryson CL, Rumsfeld JS. Medication adherence: its importance in cardiovascular outcomes. Circulation 2009;119:3028-35.
16. DeBusk RF. MULTIFIT: a new approach to risk factor modification. Cardiol Clin 1996;14:143-57.

17. Leon AC, Davis LL, Kraemer HC. The role and interpretation of pilot studies in clinical research. J Psychiatr Res 2011;45:626-9.

18. Son YJ, Lee Y, Song EK. Adherence to a sodium-restricted diet is associated with lower symptom burden and longer cardiac event-free survival in patients with heart failure. J Clin Nurs 2011;20:3029-38

19. Cook NR, Cutler JA, Obarzanek E, et al. Long term effects of dietary sodium reduction on cardiovascular disease outcomes: observational follow-up of the Trials of Hypertension Prevention (TOHP). BMJ 2007;334:885-8

20. Paterna S, Gaspare P, Fasullo $S$, et al. Normal-sodium diet compared with low-sodium diet in compensated congestive heart failure: is sodium an old enemy or a new friend? Clin Sci 2008;114:221-30.

21. O'Donnell MJ, Yusuf S, Mente A, et al. Urinary sodium and potassium excretion and risk of cardiovascular events. JAMA 2011;306:2229-38.

22. Francis GS. Notice of concern. J Card Fail 2013;19:523.

23. Gehi AK, Ali S, Na B, et al. Self-reported medication adherence and cardiovascular events in patients with stable coronary heart disease: the heart and soul study. Arch Intern Med 2007;167:1798-803. 